\title{
Die Konstruktion der Landschaft: Phänomen und Diskurs
}

\author{
N. Backhaus \\ Geographisches Institut, Universität Zürich, Winterthurerstrasse 190, 8057 Zürich, Switzerland
}

Correspondence to: N. Backhaus (norman.backhaus@geo.uzh.ch)

Leibenath, M., Heiland, S., Kilper, H., und Tzschaschel, S. (Hrsg.): Die Konstruktion der Landschaft: Phänomen und Diskurs, Wie werden Landschaften gemacht? Sozialwissenschaftliche Perspektiven auf die Konstitutierung von Kulturlandschaften, Transcript, Bielefeld, 276 S., ISBN-13: 978-3-8376-1994-2, 29,80€, 2013.

Dass Landschaften gemacht werden und je nach Perspektive, mit der man sie betrachtet unterschiedlich aussehen, dürfte kaum bestritten werden; ebenso wenig die Tatsache, dass „Landschaft" ein Wort des Alltagsgebrauchs ist, unter dem sich jede und jeder etwas vorstellt. Geht es jedoch darum, den Begriff wissenschaftlich nutzbar zu machen und zu definieren, was Landschaft ist und was nicht, wird es anspruchsvoller. Die Herausgeber haben sich dem Thema „Landschaft“ aus unterschiedlicher, aber grundsätzlich konstruktivistischer Perspektive genähert und dabei einen breiten Fächer von Anschauungen aufgespannt. Sie verstehen ihren Band als Beitrag zur Neuen Kulturgeographie, unter deren Vorzeichen auch der Projektverbund „Konstituierung von Kulturlandschaften" (KULAKon), gearbeitet hat, aus dem einige der Beiträge hervorgegangen sind. Die Konstituiertheit von Landschaften wird von Markus Leibenath im Einleitungskapitel „Konstruktivistische, interpretative Landschaftsforschung" auf ebenso klare wie eingängige Weise dargelegt. Die Konstitution der Landschaft wird in die phänomenologische und die sprachsensible Konstruktion gegliedert. Bei der ersten wird mit der Zuschreibung von Bedeutungen an Objekte der Landschaft gearbeitet (die meisten Beiträge des Bandes orientieren sich an dieser Variante des Konstruktivismus), bei der zweiten nur sprachliche Bilder verwendet und eine aussersprachliche Wirklichkeit ausgeblendet. Bei beiden Auslegungen geht es nicht darum, Objekte in der Landschaft zu entdecken, sondern mit Interpretationen zu arbeiten, was auch eine qualitative methodische Vorgehensweise nahelegt.

Bevor Beiträge mit Resultaten empirischer Arbeiten folgen, denkt Ludwig Fischer über Kulturlandschaft und Ar- beit nach. Er plädiert dabei dafür, die Arbeit an der inneren (kultur- und sozialgeschichtliche Auseinandersetzung) und äusseren (Umgestaltung durch physische Arbeit) Natur einzubeziehen, aus der die Landschaft entsteht. Er verweist dabei auf zwei Irrtümer, die es zu vermeiden gelte: die Festlegung der Landschaft als zum schönen Bild komponierte Natur und die Annahme dass aus den zivilisatorischen Erscheinungsformen unserer Umwelt die Präsenz der „Natur“ verschwunden sei.

Im Band folgen nun fünf Beiträge empirischer Landschaftsforschung. Tanja Mölders erforscht am Beispiel des Biosphärenreservats Mittelelbe Einheit und Differenz zwischen Natur- und Kulturlandschaften. Sie fragt sich, weswegen sich die Trennung beharrlich hält, wo doch weder materiell-physisch noch symbolisch-diskursiv eine Unterscheidung möglich sei, kann jedoch letztlich keine eindeutige Antwort darauf geben. Als Sollbruchstellen identifiziert sie die ästhetische und die (natur-)wissenschaftliche Deutung des Landschaftsbegriffs und erkennt, dass sich die Natur des Naturschutzes stark am Materiellen orientiert. Ökologische Begründungen zum Schutz von Landschaften werden so gegenüber symbolischen in den Vordergrund gerückt.

Gleich drei Fallbeispiele nimmt sich Monika Micheels in ihrem Beitrag zur subjektiven Konstruktion von (Kultur-)Landschaft in der Alltagspraxis vor: eine gewachsene Kulturlandschaft (Oberlausitzer Heide- und Teichlandschaft), eine urbane Kulturlandschaft (Dresdner Elbtal) und eine Bergbaufolgelandschaft (Neue Landschaft Ronneberg). Die Welt wird dabei im Sinne von Alfred Schütz als sinnhaft verstehbar konzipiert. Leider wird dabei nicht auf den Begriff der Relevanz eingegangen, mit dem einige der Erkenntnisse hätten stringenter interpretiert werden können. Zu den wichtigsten Erkenntnissen gehört, dass die Befragten mit Landschaft positive Konnotationen verbinden und damit auch Erwartungen an ihre Ausgestaltung und Entwicklung haben. Auch wenn dabei der Aspekt des Bewahrens wichtig ist, werden durchaus Neuauslegungen vorgenommen und Veränderungen (meist im Nachhinein) akzeptiert. Im Widerspruch zum handlungstheoretischen Zugang, der eingangs gewählt 
wurde, steht die knappe Aussage im Fazit, ,,dass das Individuum nicht zwangsläufig als intentional handelndes Wesen verstanden werden kann, sondern dass es Landschaft vielmehr unbewusst und als selbstverständlich wahrnimmt".

Landschaften sind nicht nur Teil des gelebten Alltags, sie werden auch geplant. Dem Planungsaspekt haben sich Wera Wojtkiewicz und Stefan Heiland zugewandt bei ihrer Untersuchung kommunaler Landschaftspläne einiger deutscher Bundesländer (die Auswahl ist ausgewogen, die Kriterien der Auswahl jedoch etwas unklar). Drei verschiedene Bedeutungszuschreibungen werden in abnehmender Wichtigkeit herausgearbeitet: die ökologische (Artenschutz, Träger eines funktionsfähigen Naturhaushalts), die soziokulturell orientierte (sinnliches Erleben, Identifikation, Schönheit) und die nutzungsorientierte (Voraussetzung für landwirtschaftliche Produktion, Wasserversorgung). Im Fazit werden aufgrund der Resultate drei kritische Fragen an die Landschaftsplanung aufgeworfen: (1) „Werden die in den Landschaftsplänen zum Ausdruck kommenden Landschaftsverständnisse den rechtlichen Vorgaben gerecht?" Eher weniger, da die Dominanz ökologisch orientierter Bedeutungszuweisungen den eigentlich bestehenden Gestaltungsspielraum zu wenig ausnützt. (2) „In welchem Verhältnis stehen landschaftsplanerische Verständnisse zu Landschaftsverständnissen von Laien?" Auch hier wäre eine verstärkte Berücksichtigung soziokultureller Bedeutungen angebracht. (3) „In welchem Verhältnis stehen landschaftsplanerische Verständnisse zu aktuellen raum- bzw. landschaftsprägenden Nutzungen und Entwicklungen?" Eine Anschlussfähigkeit an wirtschaftliche und technologische Entwicklungen ist nur bedingt vorhanden und müsste überdacht werden.

Den verschiedenen politischen Akteuren bei der Konstruktion von Kulturlandschaften - Denkmalpflege, Naturschutz und regionale Entwicklungspolitik - widmen sich Heiderose Kilper und Ludger Gailing. Dabei wird die Komplexität des Forschungsgegenstandes deutlich und auch, dass sich die Handlungsräume der Akteure zwar überlappen, doch nur schwach miteinander vernetzt sind.

Konsequent diskursiv wird die Landschaft im Beitrag von Markus Leibenath und Antje Otto zu einem Windenergieprojekt betrachtet. Klar wird die sonst nicht immer ganz einfach zu verstehende poststrukturalistische Diskurstheorie nach Laclau und Mouffe eingeführt und umgesetzt. Dabei wird geschaut, mit welchen Äquivalenzierungen bzw. Kontraritätsbeziehungen die Diskurse von Befürwortern und Gegnern des Windkraftparks verknüpft werden. Beim „Befürworterdiskurs“" werden Verbindungen zu Diskursen um Fortschritt, Klimawandel und ungelöste Endlagerungsprobleme herangezogen und der Rödeser Berg, auf dem die Windräder zu stehen kommen sollen, als Wirtschaftswald ohne besondere Schutzwürdigkeit portraitiert. Der „Gegnerdiskurs“ verbindet sich mit Begriffen wie „optischer Wahnsinn“, Naturschutz, $\mathrm{CO}_{2}$-Senke und Lebensstiländerung und zeichnet den Rödeser Berg als Wildkatzenrefugium und Naherholungsgebiet. Ideologische Verhärtungen lassen sich am beidseitigen häufigen Gebrauch es Begriffs „Katastrophe“ ablesen.

Der Band wird abgeschlossen durch ein Traktat von Olaf Kühne zu „Macht und Landschaft: Annäherungen an die Konstruktionen von Experten und Laien“. Der Beitrag greift weit aus und wartet mit verschiedenen Klassifikationen (Vertreter von Landschaftsbegriffen, Landschaftsdimensionen, Grundtypen der Macht, Stereotype der Landschaft) auf. Experten- und Laientum werden jedoch als eher rigide Gegensätze begriffen, bei der das Expertenwissen v.a. negativ konnotiert wird. Der zur Erklärung verwendete Machtbegriff wirkt mit seiner Konzentration auf Beherrschung antiquiert. So bleibt die fürsorgliche Machtausübung unerwähnt, die gerade für Fragen zur Landschaftsentwicklung interessant wäre. Des Weiteren kommt das erläuterte Demokratieverständnis ohne den Begriff Partizipation aus, was zumindest eine schweizerische Leserschaft irritieren dürfte.

Nach der klaren Einleitung und v.a. nach dem gelungenen vorletzten Beitrag wirkt der Abschluss des Bandes etwas ungelenk, was jedoch den Wert des Buches wenig beeinträchtigt. Es gibt die Breite möglicher konstruktivistischer Zugänge zur Landschaftsforschung gut wieder. Auch wird mehr oder weniger deutlich, dass eine „ökologische“, auf (natur-)wissenschaftlichen Grundlagen basierende Argumentation in vielen Diskussionen um Landschaften und ihre Entwicklung eine dominante Rolle spielt gegenüber soziokulturellen Sichtweisen. Hier leistet der Band einen wichtigen aufklärerischen Beitrag und es bleibt die Hoffnung, dass die konstruktivistische Art über Landschaft nachzudenken, nicht weiter das in der Einleitung festgestellte Nischendasein fristen muss sondern Nachahmung findet. 